\title{
Development of SyNRAC-Formula Description and New Functions
}

\author{
Hitoshi Yanami ${ }^{1,2}$ and Hirokazu Anai ${ }^{1,2}$ \\ 1 Information Technology Core Laboratories, Fujitsu Laboratories Ltd. \\ Kamikodanaka 4-1-1, Nakahara-ku, Kawasaki 211-8588, Japan \\ yanami@flab.fujitsu.co.jp, anai@jp.fujitsu.com \\ 2 CREST, Japan Science and Technology Agency \\ Kawaguchi Center Building, 4-1-8, Honcho, Kawaguchi 332-0012, Japan
}

\begin{abstract}
In this paper we present newly developed functions in Maplepackage SyNRAC, for solving real algebraic constraints derived from various engineering problems. The current version of SyNRAC provides quantifier elimination (QE) for the quadratic case and an environment dealing with first-order formulas over the reals (including new simplifiers of formulas) on Maple.
\end{abstract}

\section{Introduction}

We presented Maple-package SyNRAC for solving real algebraic constraints in 2003 1. SyNRAC stands for a Symbolic-Numeric toolbox for Real Algebraic Constraints and is aimed to be a comprehensive toolbox composed of a collection of symbolic, numeric, and symbolic-numeric solvers for real algebraic constraints derived from various engineering problems.

In this paper we show the current status of development of SyNRAC. In the previous version of SyNRAC [1] the following algorithms were available

- a special QE by the Sturm-Habicht sequence for sign definite condition,

- a special QE by virtual substitution for linear formulas,

- some naive simplifications of quantifier-free formulas.

Besides, the current version of SyNRAC provides the following:

- an environment dealing with first-order formulas over the reals,

- a special QE by virtual substitution for quadratic formulas,

- some new standard simplifiers of formulas.

Since we firstly presented SyNRAC, we have introduced some new operational symbols and fixed a notation system for expressing formulas. We are now developing our tool under the basis of the new environment. The QE algorithms previously equipped have also been reimplemented after the latest setting. These new features greatly extend the applicability and tractability of SyNRAC for solving real algebraic constraints in engineering. The current notation for first-order logic over the reals is much easier to read than the previous one. This helps users describe mathematical formulas for various types of real algebraic constraints. A 
special QE method for quadratic formulas widens the application areas of SyNRAC in actual problems (see [2]). The simplifiers can reduce the size of a given formula. This contributes not only to improve recognition of formulas but also to remarkably improve the efficiency of special QE procedures based on virtual substitution.

Furthermore, using SyNRAC as a kernel, we are now pushing the further development of design tools based on computer algebra (in particular, QE) in various application fields: One successful attempt is the development of a toolbox for parametric robust control design on MATLAB [3] based on the authors' previous works concerning QE-based robust control design [4,5,6,7].

\section{A New Environment for First-Order Formulas over the Reals}

When we say a real algebraic constraint, what we have in mind is a first-order formula over the reals. We describe what type of formulas we are dealing with and how they are expressed in SyNRAC.

An atomic formula is an equality or inequality $f\left(x_{1}, \ldots, x_{n}\right) \rho g\left(x_{1}, \ldots, x_{n}\right)$, where $f$ and $g$ are polynomials in a finite number of indeterminates over $\mathbb{Q}$ and $\rho$ is one of the relational operators $\{=, \neq, \leq,<\}$. A formula is a string obtained by appropriately arranging atomic formulas, logical operators, or existential/universal quantifiers. Here is an example of existential formulas with respect to $x, y$, and $z$

$$
\exists x \exists y \exists z\left(f_{1} \wedge f_{2} \wedge\left(h_{1} \vee h_{2}\right) \wedge f_{3}\right) \Longrightarrow \neg\left(g_{1} \wedge g_{2}\right)
$$

where $f_{i}, g_{i}$, and $h_{i}$ are atomic formulas.

To express formulas in SyNRAC, we need to prepare and fix notational symbols for $\exists, \forall, \wedge, \vee, \neg$, and so forth. In the earlier stages of implementation, we were using relational and logical operators bundled in Maple. As we proceeded, it turned out that some of the Maple's operators are unsuitable for our purpose. Let us show a simple example. Let $x$ be just an indeterminate. The evalb command, which evaluates a relation in Boolean context, in Maple returns false when $x=0$ is input. This behavior does not meet our expectation, because we want to remain $x=0$ unchanged unless $x$ is assigned a value.

To avoid such reactions, we have introduced a user-defined operator \& 1 and replaced it for the Maple's equality symbol '='. To maintain consistency, the other relational operators are redefined by adding " $\&$ " at the forefront of the respective commands (see Table 1 . Some of them are just an alias for the Maple's corresponding command. Logical operators and quantifier symbols have also been redefined in the same way as in Tables 2 and 3. In SyNRAC, the atomic formula $x^{2}-2 y^{2}-3 z^{2} \leq x y-6 y z-z+7$ is expressed in

\footnotetext{
${ }^{1}$ A Maple user can form a neutral operator symbol by using \&name (the ampersand character "\&" followed by one or more characters).
} 
Table 1. The relational operators in SyNRAC

\begin{tabular}{l} 
Operator $=\neq \leq<\geq>$ \\
\hline Notation $\&=\&<>\quad \&<=\quad \&<\quad \&>=\quad \&>$
\end{tabular}

Table 2. The logical operators in SyNRAC

\begin{tabular}{r|rrrrrr} 
Operator & $\wedge$ & $\vee$ & $\neg$ & $\Longrightarrow$ & $\Longleftrightarrow$ & $\Longleftrightarrow$ \\
\hline Notation & \&and & \&or & \&not & \&impl & \&repl & \&equiv
\end{tabular}

Table 3. The quantifiers in SyNRAC

\begin{tabular}{c|cc} 
Operator & $\exists x_{1} \cdots \exists x_{n} \varphi$ & $\forall x_{1} \cdots \forall x_{n} \varphi$ \\
\hline Notation & $\& \operatorname{Ex}\left(\left[x_{1}, \ldots, x_{n}\right], \varphi\right)$ & $\& \operatorname{All}\left(\left[x_{1}, \ldots, x_{n}\right], \varphi\right)$
\end{tabular}

$$
\left(x^{\wedge} 2-2 * y^{\wedge} 2-3 * z^{\wedge} 2\right) \quad \&<=(x * y-6 * y * z-z+7) 2
$$

The example formula above is expressed in the following:

\& $\operatorname{Ex}\left([\mathrm{x}, \mathrm{y}, \mathrm{z}],\left(f_{1}\right.\right.$ \&and $f_{2}$ \&and $\left(h_{1}\right.$ \&or $\left.h_{2}\right)$ \&and $\left.f_{3}\right)$ \&impl $\operatorname{knot}\left(g_{1}\right.$ \&and $\left.\left.g_{2}\right)\right)$.

The operators \&and and \&or can also be used as a prefix operator, taking a list of operands as an argument. The expression \&and $\left(\left[f_{1}, f_{2}, \ldots, f_{n}\right]\right)$ is equivalent in SyNRAC to $f_{1}$ \&and $f_{2}$ \&and $\cdots$ \&and $f_{n}$.

According to these notational rules, QE algorithms has been (re)implemented in SyNRAC. In addition, several basic utility functions on formulas are provided in SyNRAC, for example, functions for counting the number of atomic formulas, extracting atomic formulas from a formula as a list, and so on. Moreover, some computations for the disjunctive normal form 3 are also available.

\section{Solving Quadratic Algebraic Constraints over the Reals}

Here we briefly explain a special QE by virtual substitution of parametric test points that is applicable to formulas in which the quantified variables appear at most quadratically (see [8] for details). We call a formula whose atomic subformulas are at most quadratic (linear) with respect to its quantified variables a quadratic (linear) formula, respectively.

Let

$$
\psi\left(p_{1}, \ldots, p_{m}\right) \equiv Q_{1} x_{1} \cdots Q_{n} x_{n} \varphi\left(p_{1}, \ldots, p_{m}, x_{1}, \ldots, x_{n}\right)
$$

2 The polynomials both sides should be enclosed within parentheses since the userdefined operator \&name in Maple has higher priority than the basic arithmetic operators. In the examples in the present paper, we leave them out when too convoluted.

${ }^{3} \mathrm{~A}$ formula is called a disjunctive normal form if it is a disjunction (a sequence of $\checkmark$ 's) consisting of one or more disjuncts, each of which is a conjunction (a sequence of $\wedge$ 's) of one or more atomic formulas. 
be a linear or quadratic formula, where $Q_{i} \in\{\forall, \exists\}$ and $\varphi$ is a quantifier-free formula. By using the equivalence $\forall x \varphi(x) \Longleftrightarrow \neg(\exists x \neg \varphi(x))$, we can change the formula into an equivalent one of the form $(\neg) \exists x_{1} \cdots(\neg) \exists x_{n}(\neg) \varphi$. The negation ' $\neg$ ' that precedes a quantifier-free formula can be easily eliminated (use De Morgan's law and rewrite the atomic subformulas), which is not essential part of QE. Therefore we may focus our attention on an existential formula, i.e., a formula of the form $\exists x_{1} \ldots \exists x_{n} \varphi\left(p_{1}, \ldots, p_{m}, x_{1}, \ldots, x_{n}\right)$. Furthermore, it is sufficient to show how to eliminate $\exists x$ in $\exists x \varphi$, since all the quantifiers in the formula can be eliminated by removing one by one from the innermost one.

Now our main purpose is to eliminate the quantified variable $\exists x$ in

$$
\psi^{\prime}\left(p_{1}, \ldots, p_{m}\right) \equiv \exists x \varphi\left(p_{1}, \ldots, p_{m}, x\right)
$$

with $\varphi\left(p_{1}, \ldots, p_{m}, x\right)$ quantifier-free and quadratic, and obtain an equivalent quantifier-free formula $\psi^{\prime}\left(p_{1}, \ldots, p_{m}\right)$. For fixed real values $q_{1}, \ldots, q_{m}$ for the parameters $p_{1}, \ldots, p_{m}$, all polynomials appearing in $\varphi(x)$ are linear or quadratic. Therefore, the set $M=\left\{r \in \mathbb{R} \mid \varphi\left(q_{1}, \ldots, q_{m}, r\right)\right\}$ of real values $r$ for $x$ satisfying $\varphi$ is a finite union of closed, open, or half-open intervals over $\mathbb{R}$. The endpoints of these intervals are among $\pm \infty$ and the real zeros of atomic formulas in $\varphi$. Then candidate terms, say, $t_{1}, \ldots, t_{k}$, for those zeros can be constructed by the solution formulas for linear or quadratic equations.

If $\varphi$ does not contain any strict inequalities, all the intervals composing $M$ are either unbounded or closed. In the closed case such an interval contains its real endpoint. So $M$ is nonempty if and only if the substitution of $\pm \infty$ or of one of the candidate solutions $t_{j}$ for $x$ satisfies $\varphi$. Let $S$ be the candidate set $S=\left\{t_{1}, \ldots, t_{k}, \pm \infty\right\}$. Such a set is called an elimination set for $\exists x \varphi$. We obtain a quantifier-free formula equivalent to $\exists x \varphi$ by substituting all candidates in $S$ into $\varphi$ disjunctively:

$$
\exists x \varphi \Longleftrightarrow \bigvee_{t \in S} \varphi(x / / t)
$$

We note that there is a procedure assigning the expression $\varphi(x / t)$ obtained from $\varphi$ by substituting $t$ for $x$ an equivalent formula [8]. We denote the resulting formula by $\varphi(x / / t)$.

If $\varphi$ contains strict inequalities, we need to add to $S$ other candidates of the form $s \pm \epsilon$, where $s$ is a candidate solution for some left-hand polynomial in a strict inequality and $\epsilon$ is a positive infinitesimal.

For improving the efficiency of this method, the following two points are crucial: (i) refining the elimination set $S$ by a scrupulous selection of a smaller number of candidates in $S$; (ii) integrating with sophisticated simplifications of quantifier-free formulas. SyNRAC now employs three types of elimination sets proposed in 9]. Simplifications in SyNRAC are discussed in the next section.

Moreover, (heuristic) techniques for decreasing the degree during elimination are important for raising the applicability of quadratic QE, because after one quantifier is eliminated for a quadratic case the degree of other quantified variables may increase. Only simple degree-decreasing functions are implemented in the current version of SyNRAC. 


\section{Simplification}

In the present paper, the term simplification is used for simplification of quantifier-free formulas. When a quantifier is eliminated in a given first-order formula with a special QE procedure, its quantifier-free part usually gets larger. During a QE algorithm, formulas under manipulation tend to get extremely large, deeply nested and highly redundant. That is why simplification procedures, which equivalently change a quantifier-free formula into more concise one, are important. Utilizing simplification algorithms combined with a special QE algorithm contributes to improve not only readability of the resulting formula but efficiency of the computation.

As for simplification, Maple, on which we implement our toolbox SyNRAC, can simplify certain formulas. By using Maple's evalb command for the inequality $3<5$, the value true are obtained. But it does not work for, say, ' $x<3$ and $x<5$ '; the evalb command does nothing and just returns ' $x<3$ and $x<5$ ', not ' $x<5$ '. Dolzmann and Sturm 10 summarize the rule for simplifying such formulas, to be precise, the formula ' $f \rho_{1} 0$ and/or $g \rho_{2} 0$ ', where $f$ and $g$ differ only by a constant $c$, and $\rho_{1}$ and $\rho_{2}$ are an (in)equality. They called these laws ordering theoretical smart simplification when $c=0$, i.e., $f=g$ and additive smart simplification when $c \neq 0$, respectively.

Automatic formula simplifiers are implemented in REDLOG 4 and QEPCAD 5 (see 1310 for possible simplifications). Several simplification rules including ordering theoretical and additive smart simplification are implemented in SyNRAC, which greatly increase the efficiency of our QE commands. These rules dramatically work especially when the number of quantified variables are large.

\section{Commands in SyNRAC}

In this section we show some computational examples to illustrate how commands in SyNRAC are used 6

First, you need to load the packages:

$>$ read "synrac"; with(combinat);

You can use qe_sdc to solve the formula $\forall x>0, f(x)>0$, called the sign definite condition (SDC). The first argument of qe_sdc is polynomial $f$ and the second is the variable to be eliminated. The next example shows how to use the command to solve the problem $\forall x>0, a_{2} x^{2}+a_{1} x+a_{0}>0$,

$>$ qe_sdc $\left(a 2 * x^{\wedge} 2+a 1 * x+a 0, x\right)$;

$$
\begin{aligned}
& \left(-\mathrm{a} 0 \text { \& }<0 \text { \&and } \mathrm{a} 1 \&<0 \text { \&and }-4 * a 0+a 1^{\wedge} 2 \&<0\right) \text { \&or } \\
& \left(-\mathrm{a} 0 \text { \&< } 0 \text { \&and }-\mathrm{a} 1 \text { \&< } 0 \text { \&and }-4 * a 0+a 1^{\wedge} 2 \text { \&< } 0\right. \text { ) \&or } \\
& \text { ( }-\mathrm{a} 0 \text { \&< } 0 \text { \&and }-\mathrm{a} 1 \text { \&< } 0 \text { \&and } 4 * \mathrm{a} 0-\mathrm{a} 1^{\wedge} 2 \&<0 \text { ) }
\end{aligned}
$$

time $=0.02$, bytes $=123614$

\footnotetext{
${ }^{4}$ REDLOG is a QE package based on virtual substitution on REDUCE.

${ }^{5}$ QEPCAD is a general QE package that is applicable to all first-order formulas based on cylindrical algebraic decomposition (CAD) [1112.

${ }^{6}$ All computations were executed on a Pentium III $1 \mathrm{GHz}$ processor.
} 
By using qe_lin command, you can solve the existential linear QE problem. This command takes two arguments; the former is a list of quantified variables and the latter a quantifier-free formula. In the following example, qe_lin eliminates the two quantified variables in $\exists x \exists y(y>2 x+3 \wedge x>0 \wedge y<s)$ and returns a condition with regard to $s$.

$>$ qe_lin $(\& \operatorname{Ex}([x, y], y \&>2 * x+3$ \&and $x \&>0$ \&and $y \&<s))$;

$$
\text { time }=0.03 \text {, bytes }=144686
$$$$
-1 / 2 * s \quad \&<-3 / 2
$$

The qe_quad command can deal with quadratic QE problems. You can solve the quadratic QE problem $\exists x \exists y\left(x^{2}-4 x-5 \leq y \wedge 3 \leq x \wedge y \leq-5 s+6\right)$ as follows:

$>$ qe_quad $\left(\& \operatorname{Ex}\left([\mathrm{x}, \mathrm{y}]\right.\right.$, \&and $\left.\left.\left[\left(\mathrm{x}^{\wedge} 2-4 * \mathrm{x}-5\right) \&<=\mathrm{y}, \quad 3 \&<=\mathrm{x}, \mathrm{y} \&<=(-5 * \mathrm{~s}+6)\right]\right)\right)$;

$$
-14+5 * \mathrm{~s} \quad \&<=0
$$

time $=0.03 \mathrm{sec}$, bytes $=233514$

The two examples below show that if a decision problem is given, i.e., the input contains no free variables, each command returns the true or false value:

$>$ qe_sdc $\left(x^{\wedge} 5-x^{\wedge} 2+3 * x-9, x\right)$;

time $=1.11$, bytes $=8774262$

false

$>$ qe_lin $(\& \operatorname{Ex}([x, y], y \&<2 * x+2$ and $y \&<=-3 * x+12$ and $y \&>(1 / 3) * x+5))$;

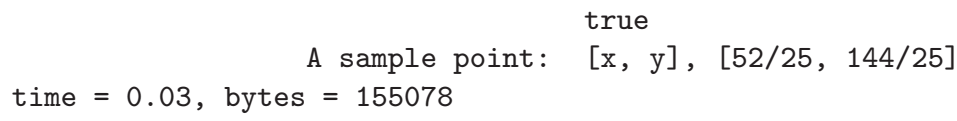

A sample point is one that makes the formula true.

By calling the qfsimple command, you can simplify quantifier-free formulas with ordering theoretical and additive smart simplification.

$>$ qfimple $((x \&<5$ \&and $x \&<c$ \&and $x \&>=10)$ \&or $(x \&<=3$ \&and $x \&<=5$ \&and $x \&>=-5$ \&and $x \&<>3)$ \&or $(x \&>7$ \&and $x \&<=d))$;

$(-3+\mathrm{x} \quad \&<=0$ \&and $-5-\mathrm{x} \&<=0)$ \&or $(-\mathrm{x} \&<-7$ \&and $-\mathrm{d}+\mathrm{x} \quad \&<=0)$ time $=0.00$, bytes $=44974$

The substsimple command simplifies quantifier-free formulas by making use of simple atomic equations. This command repeats the following two procedures: (i) solving the linear atomic equations with only one variable in each conjunctive formula and substituting its solution for the variable as far as its influence goes; (ii) calling the qfsimple command and simplifying the resulting formula. These are redone until such linear equations run out.

In the next example, $z$ in the input formula is firstly substituted by $3 / 2$ except in the 4 th atomic one, and then by using the 1st equation in the resulting formula, $x$ is replaced by $3 / 5$ in three places.

$>$ substsimple $(5 * x \&=2 * z$ \&and $9 \&>=3 * y-x$ \&and $x+4 * y+z \&>0$ \&and $2 * z-3 \&=0$ \&and $5 * x+2 * y \&<=z+3)$; 
$\mathrm{x} \&=3 / 5$ \&and $-40 * \mathrm{y} \&<21$ \&and $\mathrm{z} \&=3 / 2$ \&and $-3+4 \mathrm{y} \&<=0$ time $=0.00$, bytes $=97406$

\section{Examples}

We show two example problems from mathematical programming and solve them with SyNRAC.

Example 1 First consider the following convex quadratic programming:

minimize $x_{1}^{2}+x_{1} x_{2}+2 x_{2}^{2}$,

subject to $x_{1}+4 x_{2} \geq 16,3 x_{1}+2 x_{2} \geq 18, x_{1} \geq 0, x_{2} \geq 0$.

To obtain a description of the first-order formula, we add an unqualified variable $z$ and express the problem in

$\exists x_{1} \exists x_{2}\left(z-\left(x_{1}^{2}+x_{1} x_{2}+2 x_{2}^{2}\right) \geq 0 \wedge x_{1}+4 x_{2} \geq 16 \wedge 3 x_{1}+2 x_{2} \geq 18 \wedge x_{1} \geq 0 \wedge x_{2} \geq 0\right)$.

Eliminating the quantified variables $x_{1}$ and $x_{2}$, we can obtain a condition on $z$, from which we would obtain the range of the objective function. Quantifier elimination procedure in SyNRAC outputs the condition below in 1.78 sec:

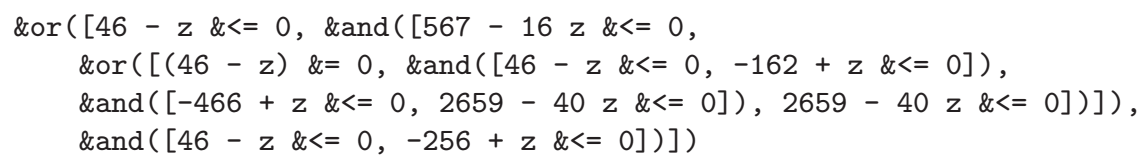

A little computation tells us that this formula is equivalent to $z \geq 46$. Thus the minimum of the objective function $x_{1}^{2}+x_{1} x_{2}+2 x_{2}^{2}$ equals 46 .

Example 2 Next we consider the following nonconvex programming:

minimize $x_{1}+3 x_{2}$,

subject to $\quad x_{1}^{2}+x_{2}-4 x_{1}-3 \geq 0, x_{1}^{2}+2 x_{2}-12 x_{1}+32 \geq 0, x_{1} \geq 0, x_{2} \geq 0$.

As in the first example, we rewrite the problem by using a slack variable $z$ into $\exists x_{1} \exists x_{2}\left(z-\left(x_{1}+3 x_{2}\right) \geq 0 \wedge x_{1}^{2}+x_{2}-4 x_{1}-3 \geq 0 \wedge x_{1}^{2}+2 x_{2}-12 x_{1}+32 \geq 0 \wedge x_{1} \geq 0 \wedge x_{2} \geq 0\right)$.

Quantifier elimination procedure as well as simplification after QE outputs the condition below in 6.12 sec:

$$
-155+25 * 42^{\sim}(1 / 2)-z \quad \&<=0
$$

Thus the minimum of the objective function $x_{1}+3 x_{2}$ is $-155+25 \sqrt{42}$, or approximately 7.02 .

\section{Conclusion}

We presented a newly developed functions in Maple-package SyNRAC. The current version of SyNRAC, in particular, provides quantifier elimination for quadratic case and some standard simplifiers of formulas over the new environment for 
first-order formulas over the reals on Maple. The new features greatly extend the applicability and tractability of SyNRAC for solving real algebraic constraints in engineering. We are continually improving the efficiency of implemented algorithms and are going to implement other algorithms (including symbolic-numeric algorithms) for solving real algebraic constraints into SyNRAC.

Now we note that based on SyNRAC the development of a toolbox for parametric robust control design on MATLAB is ongoing.

We are aware that there is still a considerable way for SyNRAC to be a sophisticated symbolic-numeric tool. Hence we will keep progressing to bridge the gap. Our goal is to develop innovative symbolic-numeric methods and to build novel design tools via SyNRAC for various fields in engineering.

Acknowledgements. The authors would like to thank Volker Weispfenning for his invaluable advice.

\section{References}

1. Anai, H., Yanami, H.: SyNRAC: A Maple-package for solving real algebraic constraints. In: Proceedings of International Workshop on Computer Algebra Systems and their Applications (CASA) 2003 (Saint Petersburg, Russian Federation), P.M.A. Sloot et al. (Eds.): ICCS 2003, LNCS 2657, Springer (2003) 828-837

2. Dolzmann, A., Sturm, T., Weispfenning, V.: Real quantifier elimination in practice. In Matzat, B.H., Greuel, G.M., Hiss, G., eds.: Algorithmic Algebra and Number Theory. Springer, Berlin (1998) 221-247

3. Sakabe, K., Yanami, H., Anai, H., Hara, S.: A MATLAB toolbox for parametric robust control system design based on symbolic computation. In: Bulletin (Kokyuroku) of RIMS (Research Institute for Mathematical Sciences, Kyoto Univ.) Workshop on Computer Algebra-Algorithms, Implementations and Applications 2003 (15-18 December 2003), (To appear)

4. Anai, H., Hara, S.: Fixed-structure robust controller synthesis based on sign definite condition by a special quantifier elimination. In: Proceedings of American Control Conference 2000. (2000) 1312-1316

5. Anai, H., Hara, S.: Linear programming approach to robust controller design by a quantifier elimination. In: Proceedings of SICE Annual Conference 2002 (Osaka, Japan). (2002) 863-869

6. Anai, H., Hara, S.: A parameter space approach for fixed-order robust controller synthesis by symbolic computation. In: Proceedings of IFAC World Congress on Automatic Control b'02. (2002)

7. Anai, H., Yanami, H., Hara, S.: SyNRAC: a maple-package for solving real algebraic constraints toward a robust parametric control toolbox. In: Proceedings of SICE Annual Conference 2003 (Fukui, Japan). (2003) 1716-1721

8. Weispfenning, V.: Quantifier elimination for real algebra - the quadratic case and beyond. Applicable Algebra in Engineering Communication and Computing 8 (1997) 85-101

9. Loos, R., Weispfenning, V.: Applying linear quantifier elimination. The Computer Journal 36 (1993) 450-462 Special issue on computational quantifier elimination.

10. Dolzmann, A., Sturm, T.: Simplification of quantifier-free formulae over ordered fields. Journal of Symbolic Computation 24 (1997) 209-231 
11. Collins, G.E.: Quantifier elimination for the elementary theory of real closed fields by cylindrical algebraic decomposition. In Brakhage, H., ed.: Automata Theory and Formal Languages. 2nd GI Conference. Volume 33 of Lecture Notes in Computer Science., Gesellschaft für Informatik, Springer-Verlag, Berlin, Heidelberg, New York (1975) 134-183

12. Collins, G.E., Hong, H.: Partial cylindrical algebraic decomposition for quantifier elimination. Journal of Symbolic Computation 12 (1991) 299-328

13. González-Vega, L.: A combinatorial algorithm solving some quantifier elimination problems. In Caviness, B., Johnson, J., eds.: Quantifier Elimination and Cylindrical Algebraic Decomposition. Texts and monographs in symbolic computation. Springer-Verlag (1998) 365-375 